\title{
Síndrome de Lemmel documentado con ecoendoscopia
}

\section{Lemmel's Syndrome Documented with Endoscopic Ultrasound}

\author{
Martín Alonso Gómez, ${ }^{+*}$ Óscar Ruiz, ${ }^{2}$ Javier Estarita. ${ }^{3}$
}

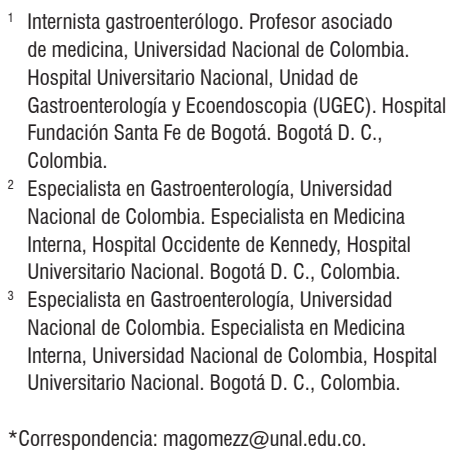

2 Especialista en Gastroenterología, Universidad Nacional de Colombia. Especialista en Medicina Interna, Hospital Occidente de Kennedy, Hospital Universitario Nacional. Bogotá D. C., Colombia.

${ }_{3}$ Especialista en Gastroenterología, Universidad Nacional de Colombia. Especialista en Medicina Interna, Universidad Nacional de Colombia, Hospital Universitario Nacional. Bogotá D. C., Colombia.

*Correspondencia: magomezz@unal.edu.co.

\begin{abstract}
Resumen
La ictericia obstructiva es una causa frecuente de consulta en los servicios de urgencias. El síndrome de Lemmel se describe como un síndrome biliar obstructivo secundario a un divertículo duodenal perpipapilar en el que se han descartado otras causas de ictericia obstructiva. Se describe un caso de ictericia obstructiva en una paciente de 84 años, en la que se realiza resonancia magnética y endosonografía biliopancreática que descartan litiasis biliar; posteriormente, es llevada a colangiopancreatografía retrógrada endoscópica (CPRE), en la que se evidencia divertículo peripapilar sin coledocolitiasis, y se realiza papilotomía, luego de la cual la paciente presenta una evolución clínica favorable.
\end{abstract}

\section{Palabras clave}

Ictericia, síndrome de Lemmel, dolor abdominal, CPRE.

\section{Abstract}

Obstructive jaundice is a frequent for patients to come to emergency services. Lemmel's syndrome is an obstructive biliary syndrome secondary to a papillary duodenal diverticulum for which other causes of obstructive jaundice have been ruled out. We describe a case of obstructive jaundice in an 84-year-old patient who underwent magnetic resonance imaging and biliopancreatic endosonography to rule out biliary lithiasis. Subsequently, the patient underwent ERCP where the papillary diverticulum was evident and without choledocholithiasis. A papillotomy was performed. Afterwards, the patient's clinical evolution was favorable.

\section{Keywords}

Jaundice, Lemmel's syndrome, abdominal pain, ERCP.

\section{PRESENTACIÓN DEL CASO}

Se presenta el caso de una paciente de 84 años con cuadro de 3 días de evolución consistente en dolor abdominal en hipocondrio derecho y epigastrio, irradiado a la espalda, asociado con náuseas, sin vómitos, por lo cual consulta a urgencias. En la valoración clínica se evidenció ictericia, sin respuesta inflamatoria sistémica, sin irritación peritoneal; en las pruebas bioquímicas se observó un patrón colestásico con hiperbilirrubinemia directa (bilirrubina total: 2,70 $\mathrm{mg} / \mathrm{dL}$, directa: $2,40 \mathrm{mg} / \mathrm{dL}$ ), con elevación de la fosfatasa alcalina (265 UI/L), discreta elevación de las aminotransferasas (alanina-aminotransferasa [ALT]: $122 \mathrm{U} / \mathrm{L}$, aspartato-aminotransferasa [AST]: 105) y ecografía abdominal con colédoco de $7 \mathrm{~mm}$, por lo que se consideró una probabilidad intermedia para coledocolitiasis y se ordenó la realización de colangiorresonancia, la cual reportó el estado postcolecistectomía y colédoco de $11 \mathrm{~mm}$, sin cálculos en su interior (Figura 1). Dado que la colangiorresonancia no estableció el diagnóstico, se decidió realizar una ultrasono- 

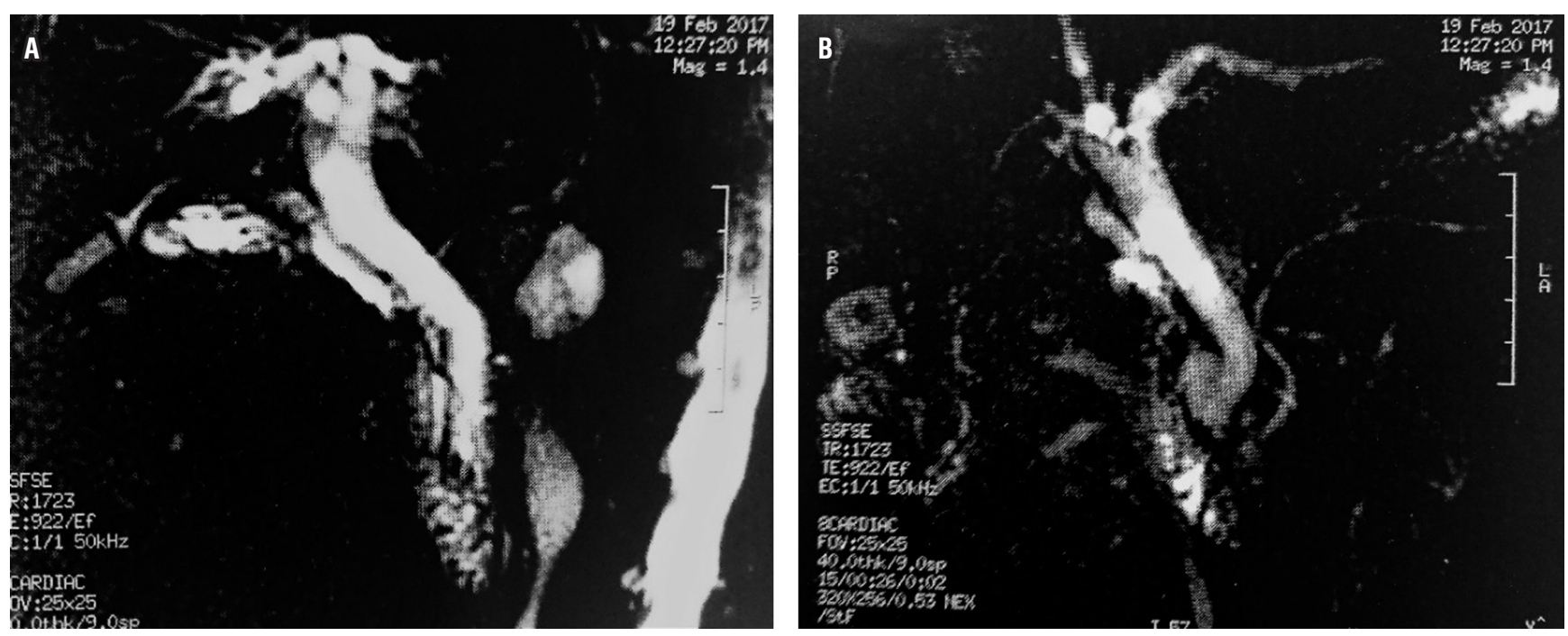

Figura 1. Colangiografía por resonancia magnética. A. Se observa vía biliar dilatada, sin defectos de llenado en su interior. B. Se observa divertículo duodenal, ausencia de vesícula biliar por antecedente quirúrgico.

grafía biliopancreática. Durante el tiempo endoscópico se evidenció un divertículo duodenal peripapilar y durante el tiempo ecográfico se evidenció la vía biliar dilatada, sin cálculos en su interior (Figura 2, Video 1).

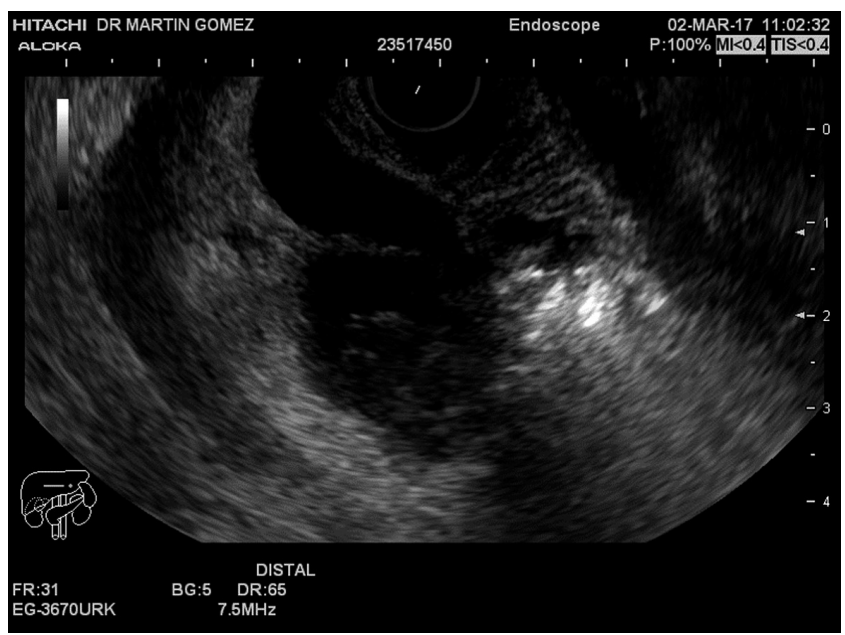

Figura 2. Ultrasonografía endoscópica biliopancreática. Se observa dilatación de la vía biliar extrahepática con divertículo duodenal que comprime la vía biliar.

Durante su estancia hospitalaria presentó persistencia de dolor abdominal con incremento en bilirrubinas, por lo que se decide realizar colangiopancreatografía retrógrada endoscópica (CPRE) diagnóstica y terapéutica. Durante la realización de CPRE se evidenció un gran divertículo peripapilar, se hizo canulación selectiva de la vía biliar y pos-

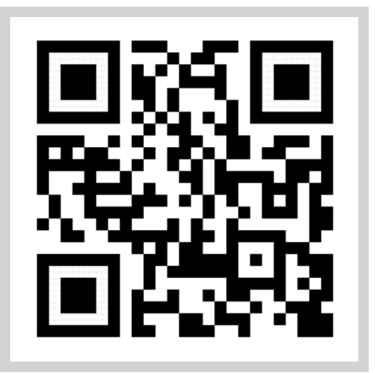

Escanee el código con su smartphone

Video 1. En la primera parte del video se muestra el tiempo endoscópico en el que se observa un gran divertículo peripapilar, corroborando el mismo en tiempo endosonográfico en la segunda parte del video, así como la presencia de detrito en su interior. https://youtu. be/1bjkFFDgCHE

terior papilotomía mediana, con abundante salida de bilis, sin barro biliar o cálculos (Figura 3).

Después de la CPRE, la paciente presentó resolución de la ictericia y de los episodios de dolor abdominal, motivo por el cual se dio de alta para continuar los estudios de manera ambulatoria. Luego de la realización de CPRE, la paciente ha presentado una evolución clínica favorable, sin nuevos episodios de dolor o ictericia.

\section{DISCUSIÓN}

La ictericia obstructiva es un motivo de consulta frecuente en los servicios de urgencias. Descrito por primera vez 

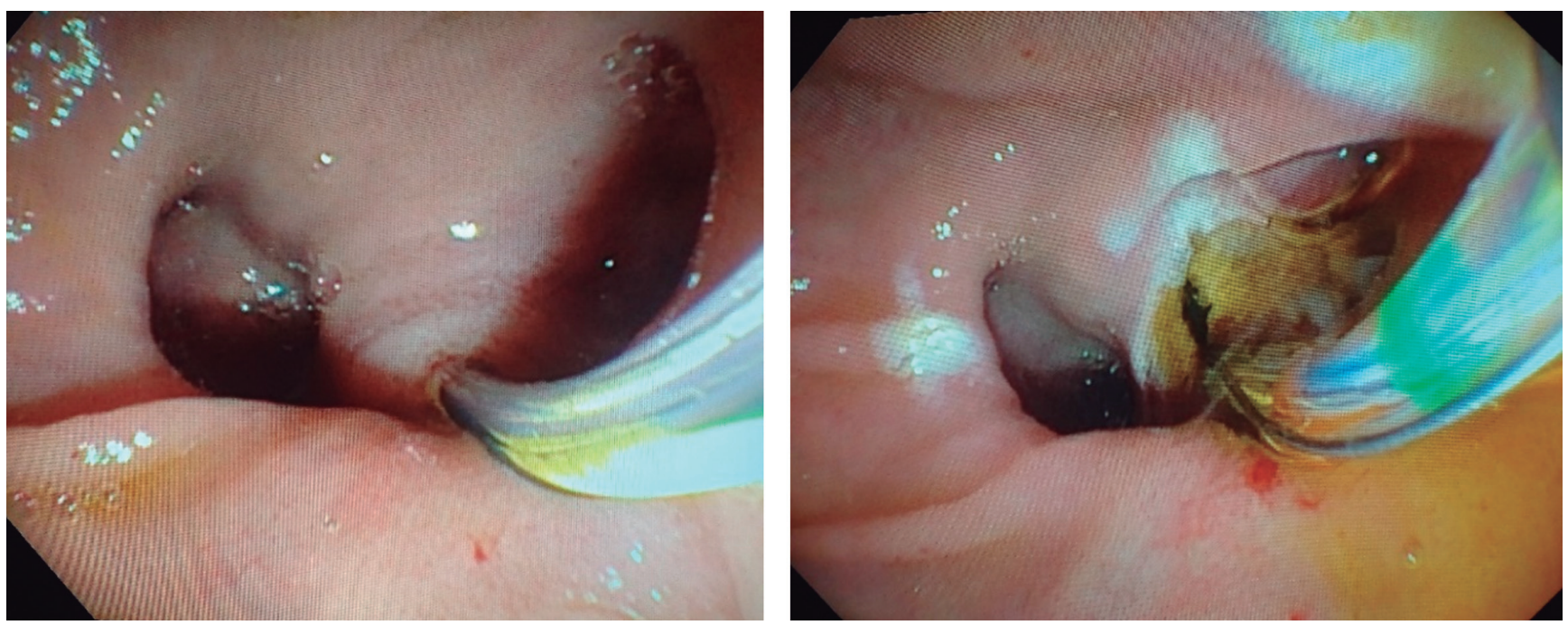

Figura 3. CPRE. A. Papila intradiverticular, canulación con papilótomo de arco. B. papilotomía mediana con salida de abundante bilis.

en 1934 por Lemmel, basado en la observación de que la presencia de un divertículo duodenal perpiapilar podría generar un síndrome biliar obstructivo (1), el síndrome de Lemmel se define como un cuadro de ictericia obstructiva en un paciente en el que se ha descartado coledocolitiasis y otras causas de obstrucción biliar y que presenta un divertículo peripapilar. El cuadro clínico asociado con el síndrome de Lemmel puede ir desde una ictericia obstructiva con dolor abdominal hasta cursar con colangitis aguda, y en ocasiones puede imitar a los tumores periampulares (2).

Debido a lo infrecuente del cuadro, no hay claridad sobre los mecanismos fisiopatológicos que llevan a la aparición de los síntomas; sin embargo, se han planteado múltiples teorías: se cree que la aparición de diverticulitis o irritación en la mecánica directa del divertículo periampular puede causar inflamación crónica de la papila, lo que posteriormente lleva a fibrosis de la misma, una condición conocida como papilitis crónica fibrosa (3). Otra teoría es la de disfunción del esfínter de Oddi secundaria a la presencia del divertículo (4). Recientemente se ha planteado como etiología probable la compresión del colédoco distal o de la ampolla por parte del divertículo periampular ocupado con un enterolito o un bezoar $(5,6)$. En el caso de nuestra paciente no se encontraron ni bezoares ni enterolitos ocupando el divertículo, pero sí se evidenció en la colangiorresonancia y en la ultrasonografía endoscópica biliopancreática un efecto compresivo del divertículo en el colédoco distal, similar a lo descrito en la literatura mundial; sin embargo, no es posible descartar la presencia de un enterolito o un bezoar previo, ya que la boca amplia del divertículo pudo haber causado la evacuación de este antes de la valoración endoscópica, lo cual además explicaría la intermitencia de los síntomas de la paciente, causales de su nueva hospitalización.

El diagnóstico del síndrome de Lemmel representa un verdadero reto, ya que es necesario descartar otras etiologías más prevalentes antes del diagnóstico; posteriormente, este se hace con la identificación del divertículo duodenal a 2 a $5 \mathrm{~cm}$ de la papila, generalmente con un duodenoscopio de visión lateral durante la CPRE. En los estudios de imágenes como la tomografía axial computarizada (TAC) o la resonancia magnética (RM), los divertículos duodenales se observan como cavitaciones en la pared duodenal, de pared delgada, situados en la segunda porción del duodeno, llenos de gas; sin embargo, en ocasiones cuando estos están llenos de líquido pueden ser confundidos con pseudoquistes, abscesos pancreáticos, adenopatías metastásicas o neoplásicas quísticas en la cabeza del páncreas $(7,8)$. En los pacientes asintomáticos no se recomienda realizar ningún tipo de manejo y en aquellos oligosintomáticos se recomienda el manejo conservador. En aquellos pacientes que cursan francamente sintomáticos, con dolor o colangitis, se podría intentar extracción o destrucción del enterolito o bezoar $(9,10)$, aunque ya que la mayoría de los casos son secundarios a papilitis crónica fibrosa o a disfunción del esfínter de Oddi, generalmente es suficiente la realización de papilotomía para el manejo de estos pacientes, como se demostró en un caso reciente publicado en la Revista Colombiana de Gastroenterología (11-13).

Aunque el síndrome de Lemmel es una entidad rara, debería considerarse en todo paciente que curse con icteri- 
cia obstructiva, en el que se hayan descartado otras causas de obstrucción, siempre que haya un divertículo periampular. El manejo del síndrome de Lemmel va a depender en gran medida de los hallazgos imagenológicos y endoscópicos, y de los síntomas presentados por el paciente, prefiriéndose el manejo endoscópico por su menor morbimortalidad que las opciones quirúrgicas.

\section{REFERENCIAS}

1. Lemmel G. Die Klinische Bedeutung der Duodenal Divertikel. Arch Verdauungskrht. 1934;56:59-70. doi: /10.1159/000196978.

2. Mallappa S, Jiao LR. Juxtapapillary duodenal diverticulum masquerading as a cystic pancreatic neoplasm. JRSM Short Rep. 2011;2(11):89. doi: 10.1258/shorts.2011.011082.

3. Manabe T, Yu GS. Duodenal diverticulum causing intermittent- persistent cholestasis. Associated with papillitis chronica fibrosa. N Y State J Med. 1977;77(13):2132-6.

4. Tomita R, Tanjoh K. Endoscopic manometry of the sphincter of Oddi in patients with Lemmel's syndrome. Surg Today. 1998;28(3):258-61. doi: 10.1007/s005950050117.

5. Rouet J, Gaujoux S, Ronot M, Palazzo M, Cauchy F, Vilgrain $\mathrm{V}$, et al. Lemmel's syndrome as a rare cause of obstructive jaundice. Clin Res Hepatol Gastroenterol. 2012;36(6):62831. doi: 10.1016/j.clinre.2012.05.002.

6. Nishida K, Kato M, Higashijima M, Takagi K, Akashi R. A case of Lemmel's syndrome caused by a large diverticular enterolith at the peripapillary portion of the duodenum. Nihon Ronen Igakkai Zasshi. 1995;32(12):825-9.
7. Macari M, Lazarus D, Israel G, Megibow A. Duodenal diverticula mimicking cystic neoplasms of the pancreas: $\mathrm{CT}$ and MR imaging findings in seven patients. AJR Am J Roentgenol. 2003;180(1):195-9. doi: 10.2214/ ajr.180.1.1800195.

8. Kim SY, Kim JN, Kwon SO, Cha IH, Ryu SH, Kim YS, et al. A case of duodenal diverticulum mimicking a peripancreatic abscess. Korean J Med, 2013;84(2):249-53. doi: 10.3904/ kjm.2013.84.2.249.

9. Nonaka T, Inamori M, Kessoku T, Ogawa $\mathrm{Y}$, Imajyo K, Yanagisawa $\mathrm{S}$, et al. Acute obstructive cholangitis caused by an enterolith in a duodenal diverticulum. Endoscopy. 2010;42 Suppl 2:E204-5. doi: 10.1055/s-0030-1255704.

10. Yoneyama F, Miyata K, Ohta H, Takeuchi E, Yamada T, Kobayashi Y. Excision of a juxtapapillary duodenal diverticulum causing biliary obstruction: report of three cases. J Hepatobiliary Pancreat Surg. 2004;11(1):69-72.10.1007/ s00534-003-0854-7.

11. Chiang TH, Lee YC, Chiu HM, Huang SP, Lin JT, Wang HP. Endoscopic therapeutics for patients with cholangitis caused by the juxtapapillary duodenal diverticulum. Hepatogastroenterology. 2006;53(70):501-5.

12. Rizwan MM, Singh H, Chandar V, Zulfiqar M, Singh V. Duodenal diverticulum and associated pancreatitis: case report with brief review of literature. World J Gastrointest Endosc. 2011;3(3):62-3. doi: 10.4253/wjge.v3.i3.62.

13. Lobo DN, Balfour TW, Iftikhar SY, Rowlands BJ. Periampullary diverticula and pancreaticobiliary disease. Br J Surg. 1999;86(5):588-97. doi: 10.1046/j.13652168.1999.01121.x. 